\title{
The value of Persian Weaning Tool in Prediction of patients' weaning outcome compared with Physician- directed approach: A Diagnostic Accuracy Study
}

\author{
Jamileh Mirzaali' ${ }^{1}$, Mohammad Ali Vakili², Homeira Khoddam ${ }^{* 3}$
}

1. School of nursing and Midwifery, GolestanUniversity of Medical Sciences, Gorgan, Iran.

2. Department of Biostatistics, School of Medicine, Golestan University of Medical Sciences, Gorgan, Iran.

3. Nursing Research Center, Golestan University of Medical Sciences, Gorgan, Iran.

*Correspondence: Nursing Research Center, Golestan University of Medical Sciences, Gorgan, Iran.Tel: +98-911 371 5485. Email: Khoddam@Goums.ac.ir

Received December 42, 2019; Accepted December13, 2019

\section{Abstract}

Background: One of the important criteria in patients receiving artificial respiration is the time of weaning from the mechanical ventilator. As physician's decision might be somehow subjective, several tools have been suggested for prediction of the time of weaning more objectively. This study aimed to determine the predictive value of Persian Weaning Tool (PWT) compared with Physician- directed approach as the gold standard.

Methods: This diagnostic accuracy study was done in 2016-2017 in Two Medical and Educational Centers of Gorgan, Iran. 97 admitted patients in intensive care units, under mechanical ventilation were evaluated. The patients were recruited into the study by a convenience sampling method and evaluated for readiness to wean using two approaches (physician's decision and using PWT). Successful weaning was considered as the ability of patient to breathe spontaneously during the first 48 hours after weaning. The sensitivity, specificity, positive predictive value (PPV), negative predictive value (NPV), positive likelihood ratio (PLR), and negative likelihood ratio (NLR), as well as the agreement (kappa coefficient) between the two approaches, were calculated. In addition, to compare the differences between variables in two groups, chi-Square, $\mathrm{T}$ and man-Whitney tests were used. All analyses were performed using SPSS software ver.16, and MedCalc program ver.13. $\mathrm{P}<0.05$ was considered as statistically significant.

Results: Most patients (64.9\%) were men. The mean age, duration of hospital admission, and 
duration of mechanical ventilation of the participants were $46.49 \pm 18.15$ years, $67.11 \pm 7.14$ days, and $31.5 \pm 2.5$ days, respectively. Weaning was successful in $87.6 \%$ of the patients. PWT had a significant agreement with the physician's choice (kappa coefficient $=0.637, \mathrm{P}<0.001$ ) with sensitivity, specificity, PPV, NPV, PLR, and NLR of 100\%, 50\%, 93.4\%, 100\%, 2, and 0, respectively. The cut-off level of 53 was considered as the best point to improve the diagnostic accuracy to $92.94 \%, 75 \%, 96.3 \%, 60 \%, 3.72$, and 0.094 , respectively.

Conclusions: Findings showed that PWT is an accurate tool for predicting the readiness of patients for weaning objectively. This tool can be used as a complementary approach by physicians and other care providers in intensive care units.

Keywords: Mechanical Ventilation weaning; Predictive Value of Tests; Persian, Tool DOI:10.29252/jgbfnm.17.1.1

\section{Introduction}

Mechanical ventilation is one of the main components of resuscitation and a fundamental medical device for critically ill patients with respiratory insufficiency requiring artificial respiration, especially in patients admitted to Intensive Care Units (ICUs) (1). Mechanical ventilators define and adjust the patient's breathing pattern using actuators, sensors, and digital electronics and hence increase patients' survival (2). However, using a mechanical ventilator can cause several complications for the patients, such as ventilator-induced lung injury, ventilator-associated pneumonia, poor nutrition, neuromyopathy, and tracheal complications (3). Therefore, it is important to appropriately select patients for using the mechanical ventilator and for weaning the patients from the ventilator to reduce patients' costs and complications (4).Weaning the patients from the mechanical ventilator is the gradual process of decreasing ventilator support, which takes about $40 \%$ of the duration of mechanical ventilation process (5). The decision of weaning process is mainly made and performed by the physician, according to the suggested criteria and clinical decision support systems (6, 7). But physician-directed weaning requiresfrequent visits by physicians that occupy physicians' time; therefore, some have suggested related protocols to be used by nurses or respiratory therapist in order to save physicians' time and enable physicians to focus on other duties that cannot be performed by non-physicians $(8,9)$. Accordingly, different scoring systems have been suggested to predict success and timely weaning of patients from the ventilator, such as the Acute Physiology and Chronic Health Evaluation II (APACHE II) and sequential organfailure assessment (SOFA) scores, Burn's Wean Assessment Program (BWAP), and Morganroth's scale; nonetheless, each of the suggested scores has its own limitations (10). Because of the limitations of the suggested scoring systems, an Iranian assessment tool has been developed by Irajpour and colleagues, entitled "Persian Weaning Tool (PWT)", in order to be used for on time weaning of the patient from the mechanical ventilator (11). Bazrafshan and colleagues have shown that PWT has acceptable validity and inter-rater reliability, in comparison with BWAP and Morganroth's scale (12). Beside the validity and reliability, it is important to evaluate whether the diagnostic power of this tool is as accurate as the physician-directed diagnosis. However, as far as we are concerned, to date, the diagnostic accuracy of this tool has not yet been evaluated. Therefore, in this study, we aimed to determine the predictive value of PWT and factors affecting patients' 
outcomes. For this purpose, we compared the patients' outcome of weaning from ventilator between physician-directed and PWT-directed approach.

\section{Methods}

In the present diagnostic accuracy study, 97 patients admitted to ICU under mechanical ventilation were evaluated. The study was performed at $5^{\text {th }}$ Azar and Shahid Sayyad Shirazi Hospitals, the two medical and educational centers of Golestan University of Medical Sciences, Gorgan, Iran with 38 ICU beds (27 and 11 ICU beds, respectively). The study duration was from November 2016 to May 2017. The study's protocol was approved by the Ethics Committee of Golestan University of Medical Sciences (code: 940430125).

The sample size was calculated using the below formula. Based on a pilot study on 22 patients admitted to the ICUs of the same hospitals, considering the sensitivity of PWT at $90 \%$, the study's confidence interval of $95 \%$, and the accuracy of $0.06,97$ patients were studied.

$$
n=z^{2} \frac{p(1-p)}{d^{2}}
$$

Accordingly, every patient who met the study's inclusion criteria were included in the study by the convenience sampling method. The inclusion criteria of this study were considered as patients' age range from 18 to 80 years, being under mechanical ventilation for at least 48 hours and less than 3 weeks, and having Endotracheal Tube (ETT). The patients with a history of chronic respiratory, cardiac, neuromuscular, or mental diseases, signs or history of sinusitis, and having received sedative drugs recently were excluded. Before enrollment of the patients into the study, the design and objectives of the study were explained to their caregivers and written informed consent was obtained from their families for the participation of their patients in the study. Any patient with accidental extubation was excluded from the study.

The ventilators that were used included Extended Air Liquid Medical Systems (made in France) and Hamilton Medical AG, C2 (made in Switzerland). The researcher recorded patients' age, sex, duration of hospital admission and mechanical ventilation, as well as the results of the physician's assessment for weaning, the patients' outcomes, and completed the PWT questionnaire. The patients who were recruited into the study were considered by the physician for weaning preparedness. The physician-based criteria for readiness of the patients for weaning from the mechanical ventilation included (13): resolution or improvement of the underlying disease, for which mechanical ventilation was initiated, no fever, arterial oxygen saturation $\left(\mathrm{O}_{2}\right.$ Sat.) $>90 \%$, while receiving oxygen, $\mathrm{Fio}_{2} \leq 0.4$, positive end-expiratory pressure (PEEP) $<8$ $\mathrm{cmH}_{2} \mathrm{O}$, hemodynamic stability without vasopressor requirement, ability to breathe spontaneously according to the results of the spontaneous breathing test (SBT), being arousable, Glasgow Coma Scale (GCS) $\geq 13$, and $\mathrm{PH}>7.2$. If the patient met these criteria, before initiating the process of weaning from the mechanical ventilator, all the patients were ventilated by pressure support ventilation (PSV) of 8-10 $\mathrm{cmH} 2 \mathrm{O}$ and PEEP of $5 \mathrm{cmH} 2 \mathrm{O}$, and the frequency/tidal volume ratio $\left(\mathrm{f} / \mathrm{V}_{\mathrm{t}}\right)$ was measured in patients before SBT. Then, spontaneous breathing was tested for 2 hours, and the patient's tolerance was evaluated during this period by the physician. If the patient was stable during this test, he/she was extubated and the weaning was considered as successful. SBT was considered as negative, if any of the following criteria was found during the test: reduced $\mathrm{O}_{2}$ saturation to $<95 \%$, reduced arterial $\mathrm{O}_{2}$ pressure. to $<60$ $\mathrm{mmHg}$, increased carbon dioxide $\left(\mathrm{CO}_{2}\right)$ to $>50 \mathrm{mmHg}$, reduced $\mathrm{PH}$ to $<7.33$ or reduction of 
$>0.07$ units in $\mathrm{PH}$, respiratory rate $(\mathrm{RR})>38$ per minute or more than $50 \%$ increase during 5 minutes, heart rate $(\mathrm{HR})>140$ beats/min, systolic blood pressure $>180$ or $<90 \mathrm{mmHg}$, agitation, disorientation, or reduced consciousness. Weaning was considered as unsuccessful, if SBT was negative, the patient required re-intubation, or expired during the first 48 hours after extubating. During this process, the information required by PWT was also recorded by the researcher simultaneously.

PWT is a tool that assesses patients' conditions with 26 items in three domains, including respiratory status ( 9 items), cardiovascular status (4 items), and general condition (13 items) of the patients. It is scored by 1 (for critical patients' condition, in which the patient needs immediate intervention), 2 (when the patient needs routine care), 3 or 4 (for appropriate patients' condition), and not applicable (in some items). The tool has five 2-point items, nineteen 3-point items and two 4-points items, resulting in a total score of 26-75 (11).

Decision about the weaning process was made by a single physician for all the patients included in this study. The researcher assessed all the admitted patients in ICU on a daily basis, irrespective of their weaning process, and the physician was unaware of the results of PWT. Finally, the results of weaning (successful or unsuccessful) were compared with the information collected using PWT to calculate the power of PWT for accurate prediction of the patients' weaning from the mechanical ventilator.

For describing the data, frequency tables were used. All the statistical analyses were performed using the statistical software IBM SPSS Statistics for Windows, version 16 (SPSS Inc., Chicago, Ill., USA) and MedCalc version 13 software. For the numeric variables between the two groups (with successful or unsuccessful weaning), first the normal distribution of the data was tested by Shapiro Wilk test. The numeric data were described by mean and standard deviation (SD) and compared between the groups using independent samples $t$ test or Mann Whitney U test, whenever the data did not have a normal distribution. $\mathrm{P}<0.05$ was considered as statistically significant. The categorical variables were compared between the groups using the Chi-square test. The sensitivity, specificity, positive predictive value (PPV) and negative predictive value (NPV), positive likelihood ratio (PLR), and negative likelihood ratio (NLR) of PWT assessment were calculated by comparing the results with the physician's decision. Also, the agreement (kappa coefficient) between the two approaches was calculated. The area under the curve (AUC) of receiver operating characteristic (ROC) curve was calculated for suggestion of the best cut-off point for PWT.

\section{Results}

In this study, 97 patients (63 men) with an age range of 17-78 years, duration of hospitalization between 3-32 days (11.67 \pm 7.14$)$ and mechanical ventilation between 3-13 days $(5.31 \pm 2.5)$ were studied.

Evaluation of the patients' consciousness level and physiological measurements during the twohour period of SBT showed they could tolerate spontaneous breathing and remained stable (table $1)$. 
Table 1. Description of patients' clinical parameters before deciding for weaning

\begin{tabular}{|l|c|c|c|}
\hline Measurements & Number & Mean (SD) & Range \\
\hline Glasgow Coma Scale & 97 & $14.01(0.97)$ & $11-15$ \\
\hline Respiratory rate (bmp) & 97 & $12.4(3.63)$ & $7-26$ \\
\hline Heart rate (bmp) & 97 & $83.16(11.68)$ & $55-99$ \\
\hline Temperature 9 $\left({ }^{\circ} \mathrm{C}\right)$ & 97 & $37.16(0.54)$ & $36.0-38.1$ \\
\hline Systolic blood pressure $(\mathrm{mmHg})$ & 97 & $111.97(14.98)$ & $80-130$ \\
\hline Diastolic blood pressure $(\mathrm{mmHg})$ & 97 & $66(6.69)$ & $60-90$ \\
\hline O2 saturation $(\%)$ & 97 & $97.14(2.21)$ & $92-100$ \\
\hline PH & 97 & $7.40(0.05)$ & $7.30-7.50$ \\
\hline
\end{tabular}

Based on the findings, $86(87.6 \%)$ patients had a successful weaning and 12 patients $(12.4 \%)$ had an unsuccessful weaning. Demographic and clinical variables were compared between the groups with successful and unsuccessful weaning, the results of which are presented in table 1. As shown in the table, weaning rate was not different according to the patients' sex (P value $=0.60$ ), the underlying disease (for which mechanical ventilation was initiated) (P value $=0.37$ ), and the cause of admission (including multiple trauma), $\mathrm{P}$ value $=0.97$; (table 1). Comparing the values of variables between the groups with successful and unsuccessful weaning showed a significant difference in the median of GCS ( $\mathrm{P}$ value $=0.008$ ), duration of hospital admission $(\mathrm{P}$ value $=0.040)$, ventilation $(\mathrm{P}$ value $=0.008)$, and the mean of PWT score $(\mathrm{P}$ value $=0.001)$ (table 2).

Table 2. The weaning success rate based on patients' demographic and clinical characteristics

\begin{tabular}{|c|c|c|c|c|}
\hline Variable & Category & Total & Successful & Unsuccessful \\
\hline \multirow[t]{2}{*}{ Sex, No. $(\%)$} & Female & $34(35.05)$ & $29(34.1)$ & $5(41.7)$ \\
\hline & Male & $63(64.95)$ & $56(65.9)$ & $7(58.3)$ \\
\hline \multirow{4}{*}{$\begin{array}{l}\text { Underlying disease, } \\
\text { No. }(\%)\end{array}$} & Cardiopulmonary & $9(9.3)$ & $7(8.2)$ & $2(16.7)$ \\
\hline & Reduced consciousness & $10(10.3)$ & $8(9.4)$ & $2(16.7)$ \\
\hline & Multiple trauma & $48(49.5)$ & $42(49.4)$ & $6(50)$ \\
\hline & Other (cancer, surgery, etc.) & $30(30.9)$ & $28(32.9)$ & $2(16.7)$ \\
\hline \multirow{2}{*}{$\begin{array}{l}\text { Reason for admis- } \\
\text { sion, No. }(\%)\end{array}$} & Multiple trauma & $48(49.5)$ & $42(43.3)$ & $6(6.2)$ \\
\hline & Other diagnoses & $49(50.5)$ & $43(44.3)$ & $6(6.2)$ \\
\hline \multicolumn{3}{|c|}{ Age (years), Mean \pm SD } & $48.42 \pm 18.61$ & $56.83 \pm 12.66$ \\
\hline \multicolumn{3}{|c|}{ Duration of hospital admission (days), Mean \pm SD } & $11.23 \pm 6.73$ & $14.75 \pm 6.89$ \\
\hline \multicolumn{3}{|c|}{ Duration of ventilation (days), Mean \pm SD } & $7.08 \pm 2.71$ & $5.07 \pm 2.41$ \\
\hline \multicolumn{3}{|c|}{ Glasgow coma Scale, Mean \pm SD } & $14.10 \pm 0.90$ & $13.33 \pm 0.88$ \\
\hline \multicolumn{3}{|c|}{ Oxygen saturation $(\%)$, Mean \pm SD } & $97.17 \pm 2.20$ & $96.91 \pm 2.31$ \\
\hline \multicolumn{3}{|c|}{ Systolic blood pressure $(\mathrm{mmHg})$, Mean \pm SD } & $111.75 \pm 15.03$ & $113.50 \pm 15.15$ \\
\hline \multicolumn{3}{|c|}{ Diastolic blood pressure $(\mathrm{mmHg})$, Mean $\pm \mathrm{SD}$} & $66.02 \pm 6.73$ & $65.83 \pm 6.68$ \\
\hline \multicolumn{3}{|c|}{ Serum hemoglobin level (mg/dl), Mean \pm SD } & $12.43 \pm 1.05$ & $12.79 \pm 1.70$ \\
\hline \multicolumn{3}{|c|}{ PWT score, Mean \pm SD } & $48.42 \pm 18.61$ & $50.58 \pm 2.90$ \\
\hline
\end{tabular}


In addition, the percentage of successful weaning cases predicted using the physician-directed method $(87.6 \%)$ and PWT (93.8\%) were significantly different $(\mathrm{P}$ value $=0.001)$. Comparing the results of the PWT assessment with the patients' outcomes showed that using PWT could lead to diagnosis of all successful cases accurately with a sensitivity of $100 \%$, but it could lead to diagnosis of only half of the cases with unsuccessful weaning, which showed a specificity of $50 \%$ for PWT. Six of the patients considered as might have successfully weaned by PWT, had unsuccessful results, indicating PPV of $93.4 \%$ and all of the patients evaluated as unsuccessful by PWT had ultimately unsuccessful weaning, indicating NPV of 100\%. PLR and NLR of PWT were 2 and 9. Kappa statistic of PWT was $0.637(\mathrm{P}<0.001)$. All in all, PWT had a diagnostic accuracy of $93.8 \%$. Based on the results of ROC curve, the best cut-off was considered at 53, which increases the sensitivity, specificity, PPV, NPV, PLR, and NLR to 92.94\%, 75\%, 96.3\%, $60 \%, 3.72$ and 0.094 , respectively (figure 1 ).

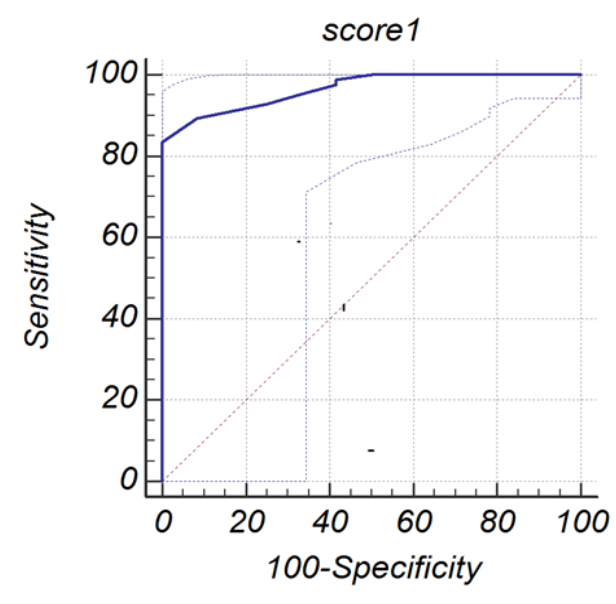

Figure 1. AUC of ROC curve for the best cut-off point of Persian Weaning Tool

\section{Discussion}

Based on the findings, $87.6 \%$ of the patients weaned by physician decision had successful outcome. Nemer and colleagues used the same criteria, entitled integrative weaning index (IWI) for evaluation of patients as that used in our study and reported a success rate of $84.7 \%$ for physician-based weaning from mechanical ventilation (14), which is close to that of the present study. This indicates the appropriateness of physician-based decision in our study. Boniatti and others modified IWI based on the $1 \mathrm{st}$ and 30thminute of the spontaneous breathing trials and compared their differences and reported a success rate of $85 \%$ (15), which is close to what reported by Nemer and colleagues, as well as ours. The slight difference between the success rates of the studies could be resulted from the different patients' characteristics. According to the evidence, several demographic, and clinical parameters can influence the patients' success rates of weaning (16). The weaning method used can also affect the failure rate (17). Boniatti and others suggested positive fluid balance and serum levels of hemoglobin and bicarbonate as predictors of weaning failure and re- 
intubation (15), which is contrary to the results of our study, as we found no significant difference between the patients with successful and failed weaning in terms of Sat.O2, blood pressure, serum levels of hemoglobin, and $\mathrm{PH}$, while the only significant variables were presented as mean GCS, duration of hospital admission, ventilation, and PWT score, and the patients with unsuccessful results had a lower GCS, higher duration of hospital stay and ventilation, as well as lower scores of PWT. In the study by $\mathrm{Wu}$ and colleagues, the duration of hospital admissionand higher modified GCS score were reported as the significant predictors of weaning failure (18), which confirm the results of the present study, while they reported success rate of weaning at $56 \%$, which is much lower than that in the present study (18). In the meantime, the patients' condition is an important factor for the weaning outcome. Not only the physical parameters (19), but also the psychological parameters, such as patients' confidence and subjective perception of autonomous breathing can also affect the weaning success rate (20). Therefore, the numerous factors affecting weaning success can be the main reason underlying the different weaning failure/success rates among the results of studies.As reported, about $40 \%$ of patients under mechanical ventilation experience difficult weaning, and weaning process decided by the physician is considered as a time-consuming process, subject to inter-rater and other types of biases (21). Accordingly, several protocols and scoring systems have been suggested for prediction of successful weaning from the mechanical ventilator to be used by non-physician technicians to save the physicians' time $(10,22)$; however, they fail to predict successful weaning, especially in patients with prolonged ventilation who display poor compliance (23, 24). Several studies have compared the success rate of weaning based on physician-based decision with automated Smart Care ${ }^{\mathrm{TM}}$ (DrägerMedical, Lübeck, Germany). The results suggest that this automated system reduced the total duration of ventilation, weaning, and hospital stay, but did not influence mortality or adverse events, specifically re-intubation (25-27). In this method, the ventilator is switched to Smart Care, and the ventilator adjusts the pressure, uses automatic weaning strategy, and executes an automatic weaning test (28); however, this tool is not available in all centers. Therefore, scoring systems have been designed to calculate and predictthe risk of successful weaning from mechanical ventilator by a non-physician staff.In the present study, the results of physician-based decision for weaning from the mechanical ventilation were evaluated with the scoring system of PWT, and the results showed a diagnostic accuracy of $93.4 \%$, a Kappa coefficient of 0.637 , which means that PWT was compatible with the conventional method in $64 \%$ of cases with a significant difference between the results of these two methods. Calculation of diagnostic accuracy of PWT showed sensitivity, specificity, PPV, NPV, PLR, and NLR of 100\%, 50\%, 93.4\%, 100\%, 2, and 0 , respectively, for this scale. PWT is an Iranian scale designed by Irajpour and colleagues to facilitate weaning of patients from mechanical ventilator (11). By reviewing the available literature about the scoring systems, Irajpour and colleagues suggested that each of the suggested scores had several limitations, and thus developed PWT for precise determination of the weaning process and claimed that this toolwas a comprehensive tool for this purpose, while its preciseness had to be compared with other tools (11). Following their study, Bazrafshan and colleagues compared the results of PWT with BWAP and Morgan Roth'sscale and suggested that PWT had acceptablevalidity and inter-rater reliability (12). In their study, they suggested cut-off point of 57 as the minimum acceptable PWT (12), while in our study, we calculated 53 as the most appropriate cut-off based on the ROC curve. Also, Bazrafshan and colleagues reported a sensitivity of $67.9 \%$ and specificity of $\wedge \mathrm{J}$ Res Dev Nurs 
Midw,Volume 17, Number 1, January, 200280.4\% for PWT (12), while we reported sensitivity and specificity of PWT at $100 \%$ and $50 \%$, respectively, which changed to $92.94 \%$ and $75 \%$, respectively, by considering the cut-off point at 53 . This difference between the results of our study and that reported by Bazrafshan and colleagues could be resulted from the fact that we compared the results of PWT evaluation with physician-based decision, while Bazrafshan and colleagues compared the results of PWT withBWAP and Morgan Roth'sscale, which we consider as a limitation of this study, as none of these scores are considered as the gold standard, and they also have several limitations and problems, which can affect the results, while we compared the results with the physician's decision, which is the routine care suggested by guidelines. We further investigated the PPV, NPV, PLR, and NLR of the test. PLR of 2 showed that PWT can predict the successful results of weaning twice more accurate in patients with final successful results, compared with those with ultimate failed results of weaning, and NLR of 0 showed that the prediction power of failed weaning in patients with ultimate results of successful weaning was 0 . The extent at which PLR is more than 1 and theextent at which NLR approaches 0 and is below 0.1 shows the superiority of the tool for the diagnosis of positive cases. Changing the cut-off point to 53 can even increase the diagnostic accuracy of the test more. Although the validity and diagnostic accuracy of other indices, such as IWI has been proven in Iranian population (29). The diagnostic accuracy of PWT has not been evaluated previously. Therefore, further studies are required to indicate the superiority of PWT to other tools and scoring systems. The present study was the first to evaluate PWT's diagnostic accuracy, compared with the physicianbased protocol, considered as the gold standard. Nevertheless, this study had some limitations, including non-randomized patients' recruitments into the study, as well as the small sample size, and lack of follow-up. Furthermore, we considered several inclusion criteria to specify the results in that population and reduce the effect of confounders on the results; therefore, the results of the study cannot easily be generalized to other subgroups of patients, such as patients with prolonged artificial respiration.

\section{Discussion}

The results of the present study showed that PWT had a high diagnostic accuracy in predicting the readiness of patients for weaning from the mechanical ventilation and had a high compatibility with the physician-based decisions for predicting the outcome of weaning. Using this method can successfully diagnose patients with successful/failed weaning results with a high sensitivity and its use can prevent the unnecessary prolongation of artificial respiration and reduce its complications. Therefore, we suggest this tool to be used by non-physicians, as an alternative or beside the physician's decision, in order to indicate the preparedness of patients for weaning from the mechanical ventilator, facilitate weaning process, reduce the duration of artificial respiration, and improve the patients' outcome. It has to be considered that PWT was scored by the study's researcher in the present study; therefore, it is necessary to investigate the diagnostic accuracy of PWT, when assessed by non-physicians, such as nurses, study the patients' long-term outcomes, and evaluate the diagnostic accuracy of PWT compared with other scoring systems. 


\section{Acknowledgements}

The present study was extracted from the results of a thesis conducted for the fulfillment of Master of Science in intensive care nursing at the school of nursing and midwifery of Golestan University of Medical Science with grant number 940714174 .It was financially supported by the Research and Technology Deputy of Golestan University of Medical Sciences, Gorgan, Iran. The authors of the present study sincerely thank the Deputy of Research and Technology of Golestan University of Medical Sciences for supporting this research.

\section{References}

1. Slutsky AS. History of mechanical ventilation. From Vesalius to ventilator-induced lung injury. Am J Respir Crit Care Med. 2015; 191(10):1106-15. [DOI:10.1164/rccm.201503-0421PP]

2. Dellaca RL, Veneroni C, Farre' R. Trends in mechanical ventilation: are weventilating our patients in the best possible way? Breathe. 2017; 13(2):84-98. [DOI:10.1183/20734735.007817]

3. Klompas M, Khan Y, Kleinman K, Evans RS, Lloyd JF, Stevenson K, et al. Multicenter evaluation of a novel surveillance paradigm for complications of mechanical ventilation. PLoSOne. 2011; 6(3):e18062. [DOI:10.1371/journal.pone.0018062]

4. Sellares J, Ferrer M, Cano E, Loureiro H, Valencia M, Torres A. Predictors of prolonged weaning and survival during ventilator weaning in a respiratory ICU. Intensive Care Med. 2011; 37(5):775-84. [DOI: 10.1007/s00134-011-2179-3]

5. Zein H, Baratloo A, Negida A, Safari S. Ventilator weaning and spontaneous breathing trials; an educational review. Emergency. 2016; 4(2):65-71.

6. MacIntyre NR. Evidence-based assessments in the ventilator discontinuation process. Respir Care. 2012; 57(10):1611-8. [DOI:10.4187/respcare.02055]

7. Hsu J-C, Chen Y-F, Chung W-S, Tan T-H, Chen T, Chiang JY. Clinical verification of a clinical decision support system for ventilator weaning. Biomed Eng Online. 2013; 12:S4. [DOI: $\underline{10.1186 / 1475-925 X-12-S 1-S 4]}$

8. Chaiwat O, Sarima N, Niyompanitpattana K, Komoltri C, Udomphorn Y, Kongsayreepong S. Protocol-directed vs. Physician-directed weaning from ventilator in intra-abdominal surgical patients. J Med Assoc Thai. 2010; 93(8):930-6.

9. Danckers M, Grosu H, Jean R, Cruz RB, Fidellaga A, Han Q, et al. Nurse-driven, protocoldirected weaning from mechanical ventilation improvesclinical outcomes and is well accepted by intensive care unit physicians. J Crit Care. 2013; 28(4):433-41. [DOI:10.1016/j.jcrc.2012.10.012]

10. Dehghani A, Abdeyazdan G, Davaridolatabadi E. An overview of the predictor standard tools for patient weaning from mechanical ventilation. Electronic physician. 2016; 8(2):1955. [DOI: $\underline{10.19082 / 1955]}$ 
11. Irajpour A, Khodaee M, Yazdannik A, Abbasi S. Developing a readiness assessment tool for weaning patients under mechanical ventilation. Iran J Nurs Midwifery Res. 2014; 19(3):273-8.

12. Bazrafshan F, Irajpour A, Abbasi S, Mahaki B.Validity and reliability of "Persian Weaning Tool" in mechanically ventilated patients. Adv Biomed Res. 2016; 5:157 [DOI:10.4103/22779175.188488]

13. Ebrahimabadi S, Moghadam AB, Vakili M, Modanloo M, Khoddam H. Studying the power of the integrative weaning index in predicting the success rate of the spontaneous breathing trial in patients under mechanical ventilation. Indian J Crit Care Med. 2017; 21(8):488-493. [DOI:10.4103/ijccm.IJCCM_10_17]

14. Nemer SN, Barbas CS, Caldeira JB, Cárias TC, Santos RG, Almeida LC, et al. A new integrative weaning index of discontinuation from mechanical ventilation. Critical Care. 2009; 13(5):R152. [DOI: 10.1186/cc8051]

15. Boniatti VM, Boniatti MM, Andrade CF, Zigiotto CC, Kaminski P, Gomes SP, et al. The modified integrative weaning index as a predictor of extubation failure. Respir Care. 2014; 59(7):1042-7. [DOI:10.4187/respcare.02652]

16. Monaco F, Drummond G, Ramsay P, Servillo G, Walsh T. Do simple ventilation and gas exchange measurements predict early successful weaning from respiratory support in unselected general intensive care patients? Br J Anaesth. 2010; 105(3):326-33. [DOI:10.1093/bja/aeq184]

17. Mabrouk AA, Mansour OF, El-Aziz AAA, Elhabashy MM, Alasdoudy AA. Evaluation of some predictors for successful weaning from mechanical ventilation. Egyptian Journal of Chest Diseases and Tuberculosis. 2015; 64(3):703-7. [DOI:10.1016/j.ejcdt.2015.03.021]

18. Wu YK, Kao KC, Hsu KH, Hsieh MJ, Tsai YH. Predictors of successful weaning from prolonged mechanical ventilation in Taiwan. Respir Med. 2009; 103(8):1189-95. [DOI:10.1016/j.rmed.2009.02.005]

19. Savi A, Teixeira C, Silva JM, Borges LG, Pereira PA, Pinto KB, et al. weaning predictors do not predict extubation failure in simple-to-wean patients. J Crit Care. 2012; 27(2):221. E1-. e8. [DOI:10.1016/j.jcrc.2011.07.079]

20. Perren A, Previsdomini M, Llamas M, Cerutti B, Györik S, Merlani G, et al. Patients' prediction of extubation success. Intensive Care Med. 2010; 36(12):2045-52. [DOI: 10.1007/s00134-010-1984-4]

21. Penuelas O, Frutos-Vivar F, Fernandez C, Anzueto A, Epstein SK, Apezteguia C, et al. Characteristics and outcomes of ventilated patients according to time to liberation from mechanical ventilation. Am J Respir Crit Care Med. 2011; 184(4):430-7. [DOI:10.1164/rccm.201011-18870C]

22. Blackwood B, Alderdice F, Burns KE, Cardwell CR, Lavery G, O'Halloran P. Protocolized 
versus non-protocolized weaning for reducing the duration of mechanical ventilation in critically ill adult patients. Cochrane Database Syst Rev. 2010; (5):Cd006904. [DOI:10.1002/14651858.CD006904.pub2]

23. Rojek-Jarmuła A, Hombach R, Krzych ŁJ. APACHE II score cannot predict successful weaning from prolonged mechanical ventilation. Chronic respiratory disease. 2017; 14(13):2705. [DOI: $10.1177 / 1479972316687100]$

24. West RR, Saidi M, Dawson D. Protocol-driven weaning from mechanical ventilation: a study into adherence and outcomes. Critical Care. 2009; 13(1):P24. [DOI: 10.1186/cc7188]

25. Turan Inal M, Memiş D, Yildirim İ. Comparıson of extubatıon tımes between protocolızed versus automated weanıng systems after major surgery in the intensive care unit. Signa vitae: journal for intesive care and emergency medicine. 2012; 7(1):23-7. [DOI:10.22514/SV71.042012.4]

26. Rose L,Schultz MJ, Cardwell CR, Jouvet P, McAuley DF, Blackwood B. Automated versus non-automated weaning for reducing the duration of mechanical ventilation for critically ill adults and children: a cochrane systematic review and meta-analysis. Crit Care. 2015; 19:48. [DOI: 10.1186/s13054-015-0755-6]

27. Burns KE, Lellouche F, Nisenbaum R, Lessard MR, Friedrich JO. Automated weaning and SBT systems versus non- automated weaning strategies for weaning time in invasively ventilated critically ill adults. Cochrane Database of Systematic Reviews. 2014. [DOI:10.1002/14651858.CD008638.pub2]

28. Kataoka G, Murai N, Kodera K, Sasaki A, Asano R, Ikeda M, et al. Clinical experience with Smart Care after off-pump coronary artery bypass for early extubation. J Artif Organs. 2007; 10(4):218-22. [DOI: 10.1007/s10047-007-0392-1]

29. Madani SJ, Saghafinia M, Nezhad HS, Ebadi A, Ghochani A, Tavasoli AF, et al. Validity of integrative weaning index of discontinuation from mechanical ventilation in Iranian ICUs. Thrita. 2013; 2:62-8. [DOI:10.5812/thrita.12827]

\section{Bibliographic information of this paper for citing:}

Mirzaali J, Vakili MA, Khoddam H. The value of Persian Weaning Tool in Prediction of patients' weaning outcome compared with Physician- directed approach: A Diagnostic Accuracy Study.

J Res Dev Nurs Midw, 2020; 17(1): 1-11.

Copyright ( 9 2020, Jamileh Mirzaali, MohammadaliVakili, Homeira Khoddam. 